\title{
Androgénothérapie en reproduction humaine
}

\author{
Frank COMHAIRE * \\ Hôpital Universitaire de Gand Département d'Endocrinologie De Pintelaan, \\ 185 B-9000 Gand, Belgique Fax: +32 91403897
}

\section{INTRODUCTION}

La testostérone est sécrétée par les cellules de Leydig dans le liquide interstitiel, et se diffuse immédiatement à travers l'épithélium spermatogénétique. Sous l'influence de la 5-alpharéductase produite par les cellules de Sertoli, la testostérone est réduite en 5-alpha-dihydrotestostérone. En suite, celle-ci est métabolisée en 5-alpha,3-alpha- androstanediol sous l'influence de la hydroxystéroïde- déhydrogenase. La concentration de ces androgènes dans le liquide interstitiel et intratubulaire est similaire. Cette concentration est environ 100 fois plus élevée que celle dans le sang périphérique, et elle est très variable. En effet, la sécrétion par les cellules de Leydig est pulsatoire puisqu'elle suit la pulsatilité de la LH. Les cellules de Sertoli sont donc exposées à des concentrations variables de testostérone. Il est possible qu'une concentration d'androgènes intratesticulaires élevée et pulsatile soit requise pour que la spermatogénèse puisse se dérouler de façon optimale, aussi bien du point de vue qualitatif que du point de vue quantitatif.

Lorsque la spermatogénèse est déficiente, celleci peut être stimulée par des traitements visant à augmenter la sécrétion testiculaire des androgènes, ou par l'administration d'androgènes exogènes.

*présenté à la lère journée scientifique de la S.A.L.F., Tours, 18 juin 1993

\section{EFFET HORMONAL DU TRAITEMENT PAR GONADOTROPINES ET PAR ANTIOESTROGENES}

L'administration de la Gonadotropine Chorionique Humaine par bolus intraveineux provoque une augmentation de la concentration de testostérone dans le sang périphérique, qui est accompagnée d'une augmentation de la concentration de l'inhibine. Cette dernière se manifeste déjà $18 \mathrm{~h}$ après l'injection, et elle est probablement induite par l'augmentation de la testostérone intratesticulaire. L'augmentation de la testostérone périphérique persiste pendant 48 et $72 \mathrm{~h}$, tandis que la concentration élevée de l'inhibine n'est pas maintenue. L'injection intraveineuse d'un bolus de FSH pure ne semble pas influencer la concentration de la testostérone dans le sang périphérique, mais elle induit une augmentation importante de la concentration de l'inhibine qui est enregistrée 48 et $72 \mathrm{~h}$ après l'injection.

La FSH semble donc stimuler la production et la sécrétion de l'inhibine par les cellules de Sertoli, sans pour autant interférer avec la sécrétion des androgènes. Ces données démontrent la dualité de la régulation de la fonction des cellules de Sertoli, et confirment l'effet stimulant des androgènes sur leur sécrétion. L'administration par voie orale de Tamoxiphène, un antioestrogène spécifique virtuellement dépourvu d'action oestrogénique intrinsèque, augmente la concentration de la testostérone mais pas celle de la $\mathrm{LH}$, et induit 
une augmentation modérée de la FSH sans augmenter le taux de l'inhibine.

\section{ANDROGÉNOTHÉRAPIE}

L'administration d'androgènes pour le traitement de la déficience spermatogénétique (idiopathique) vise à augmenter la concentration intratesticulaire de la testostérone ou de ses dérivés, dans l'espoir d'optimaliser la fonction de l'épithélium spermatogénétique. Ceci pourrait s'effectuer par l'intermédiaire d'une activité améliorée des cellules de Sertoli, mais également de façon directe sur le processus de la spermiogénèse qui semble être androgéno-dépendant. En outre, l'administration d'androgènes pourrait améliorer la fonction de l'épididyme qui dépend également d'une concentration élevée d'androgènes, en particulier de la 5- alpha-dihydrotestostérone. Il est cependant impératif que les androgènes administrés ne suppriment pas la sécrétion endogène des gonadotropines.

\section{Le traitement à forte dose de testostérone}

Par exemple l'oenantate de testostérone, (Testoviron ${ }^{\circledR}$ ) par voie intramusculaire, induit une augmentation importante, voire supraphysiologique de la concentration de testostérone dans le sang périphérique, qui entraîne une suppression immédiate de la sécrétion des gonadotropines. Celle-ci se traduit par une suppression de la spermatogénèse. Heller et collaborateurs ont suggéré qu'un "phénomène de rebond" se produirait après l'arrêt de l'administration de fortes doses de testostérone. Le traitement par rebond visait à obtenir une réaction spermatogénétique accrue qui, après une phase de "repos", dépasserait la situation basale. Cependant, il s'est avéré que le phénomène de rebond ne se produit pas chez certains malades souffrant d'oligozoospermie idiopathique. En outre, l'état de suppression de la spermatogénèse peut même persister. Ainsi l'oligozoospermie pourrait se maintenir et être plus sévère après le traitement. C'est pour cette raison que le traitement par rebond a largement été abandonné.

\section{Traitement par les dérivés de la testostérone.}

L'industrie pharmaceutique a donc créé des dérivés de la testostérone qui présentent deux caractéristiques importantes à savoir :

- leur résorption après administration par voie orale

- leur métabolisme (préférentiel) en androgènes 5-alpha réduits, au lieu de l'aromatisation.

Grâce à ces caractéristiques, ces produits devraient exercer un moindre effet suppressif sur la sécrétion de la LHRH et des gonadotropines endogènes.

La Mestérolone (Proviron ${ }^{\circledR}$ ) et l'undécanoate de la testostérone (Andriol ${ }^{\circledR}$ ) sont de tels produits.

\section{a) La Mestérolone :}

Elle est utilisée pour le traitement de l'oligozoospermie idiopathique depuis une dizaine d'années. Le résultat d'études pilotes ouvertes, donc non contrôlées, semblait être suffisamment encourageant pour justifier une étude prospective en double aveugle. Celle-ci fut menée par l'OMS, et elle a comparé l'effet d'un traitement par Mestérolone $150 \mathrm{mg} /$ jour ou $75 \mathrm{mg} / \mathrm{j}$ our, à celui d'un placebo. Cette étude n'a révélé aucune différence significative, quoiqu'une tendance favorable semblait se dessiner en faveur du traitement à dose élevée. La Mestérolone à $150 \mathrm{mg} /$ jour semblait légèrement augmenter la probabilité de grossesses. Dans une étude complémentaire, effectuée par le groupe de Gerris et nousmêmes, l'effet de $150 \mathrm{mg} /$ jour de Mestérolone fut comparé de façon double aveugle à un placebo, pendant une période de traitement de 12 mois. Les résultats de cette étude furent négatifs, puisque la probabilité de conception était inférieure dans le groupe verum que dans le groupe placebo. Il est apparu que la dose de $150 \mathrm{mg} /$ jour provoquait une suppression (partielle) de la fonction hypothalamo-hypophysaire, qui semblait inhiber l'effet favorable temporaire du placebo sur la concentration de spermatozoïdes. 


\section{b) Undécanoate de la testostérone (Andriol)}

Une étude en double aveugle menée chez 20 patients souffrant d'oligozoospermie idiopathique fut également pratiquée avec une dose forte d'Andriol ( $240 \mathrm{mg} / \mathrm{jour}$ ). Cette étude a révélé un effet positif sur la concentration du triphosphate d'adénosine dans l'éjaculat sous traitement par Andriol.

Cependant, il n'y a pas eu d'amélioration significative dans les caractéristiques conventionnelles des spermatozoïdes.

Enfin, une étude prospective, non contrôlée, sur un nombre limité de cas, a montré l'effet favorable d'un traitement de trois mois avec une dose modérée d'Andriol ( $120 \mathrm{mg} /$ jour) sur le pouvoir fécondant des spermatozoïdes in vitro. Actuellement, une étude en double aveugle est en cours dans six centres, pour essayer de confirmer cet effet thérapeutique dans la fécondation in vitro.

\section{CONCLUSIONS}

Jusqu'à présent aucun traitement hormonal n'a prouvé son efficacité dans des études en double aveugle lorsque la probabilité de conception "naturelle" est acceptée comme paramètre. Il en est de même en ce qui concerne les différentes modalités de traitement aux androgènes. Ceci n'exclut pourtant pas la possibilité d'un effet biologique sur la qualité des spermatozoïdes, qui pourrait s'exprimer par un meilleur pouvoir fécondant lors de la fécondation in vitro. D'ailleurs, il reste possible que certains androgènes aient un effet thérapeutique supérieur dans certains cas bien définis. L'étude systématique de la réponse des cellules de Sertoli à l'aide du dosage de l'inhibine dans le sang périphérique, pourrait éventuellement faciliter l'identification de ces cas particuliers. 